\title{
Resolution of Pain and Predictors of Postoperative Opioid use after Bridge-Enhanced Anterior Cruciate Ligament Repair and Anterior Cruciate Ligament Reconstruction
}

\author{
Samuel Barnett, M.D., Martha M. Murray, M.D., \\ Shanshan Liu, M.S., M.P.H., The BEAR Trial Team*, and Lyle J. Micheli, M.D.
}

Purpose: To compare postoperative pain scores and opioid use between patients undergoing a standard arthroscopic anterior cruciate ligament reconstruction (ACLR) using hamstring autograft with those undergoing a suture repair augmented with an extracellular matrix scaffold (bridge-enhanced ACL repair) performed through an arthrotomy and to determine factors predictive of postoperative opioid use and levels of overprescription. Methods: A nonrandomized controlled trial was conducted with 20 patients (10 ACLR, 10 bridge-enhanced ACL repair), aged 18 to 35 years. All surgeries were performed by a single surgeon. A pain medication log was provided to patients on discharge. No regional anesthesia was performed. Pain scores via a visual analog pain scale were recorded at each visit. Correlations between preoperative and intraoperative characteristics and postoperative opioid use were determined. Results: The total morphine-equivalent dose ranged from 30 to $309 \mathrm{mg}$ (4-42 pills oxycodone) for the ACLR group and 75 to $254 \mathrm{mg}$ (10-34 pills oxycodone) for the bridge-enhanced ACL repair group. The average opioid use per day was $35.8 \mathrm{mg}$ for the patients undergoing bridge-enhanced ACL repair and $44.2 \mathrm{mg}$ for patients undergoing ACLR $(P=.29)$. Pain scores at time points up to 2 years postoperatively were not significantly different between the 2 groups. Across both groups, the average oversupply of oxycodone was 46 pills per patient, a greater than $70 \%$ unused opiate rate. Preoperative body mass index and preoperative Knee Injury and Osteoarthritis Outcome Scores pain score were predictive of greater postoperative opioid use per day, whereas age, concurrent meniscal repair, and operative time were not. Conclusions: Total overall opiate intake was not different between the patients undergoing bridge-enhanced ACL repair through an arthrotomy and those undergoing arthroscopic ACLR. Both groups had similar pain scores from 2 weeks to 2 years postoperatively.

From the Division of Sports Medicine, Department of Orthopaedic Surgery (S.B., M.M.M., L.M., R.H., C.F., B.T., B.P., D.K., Y.-M.Y.) and Institutional Centers for Clinical and Translational Research (S.L.), Boston Children's Hospital Boston, Massachusetts, U.S.A.

The authors report the following potential conflicts of interest or sources of funding: Funding support from the Translational Research Program at Boston Children's Hospital, the Children's Hospital Orthopaedic Surgery Foundation, the Children's Hospital Sports Medicine Foundation, and the National Institutes of Health and the National Institute of Arthritis and Musculoskeletal and Skin Diseases through grant numbers R01-AR065462 and R01AR056834. This research also was conducted with support from the Football Players Health Study at Harvard University. The Football Players Health Study is funded by a grant from the National Football League Players Association. The content is solely the responsibility of the authors and does not necessarily represent the official views of Harvard Medical School, Harvard University or its affiliated academic health care centers, the National Football League Players Association, Boston Children's Hospital or the National Institutes of Health. B.T. reports personal fees from Miach Orthopaedics, outside the submitted work. D.K. reports other from Kairos Surgical, outside the submitted work. M.M.M. reports grants from National Football League Players Association, grants from Translational Research Program at $B C H$, grants from National Institutes of Health, other from Children's Orthopaedic Surgery Foundation, other from Children's Sports Medicine Foundation, during the conduct of the study; other from Miach Orthopaedics, Inc., outside the submitted work; In addition, M.M.M. has a patent owned by Boston Children's Hospital (multiple) on which she is a named inventor and which have been licensed to Miach Orthopaedics. B.P. reports personal fees from MIACH Orthopaedics, outside the submitted work. Full ICMJE author disclosure forms are available for this article online, as supplementary material.

*The BEAR Trial Team: Rachael Henderson, Christina Freiberger, Bethany Trainor, Benedikt Proffen, Dennis Kramer, Yi-Meng Yen.

Received September 6, 2019; accepted February 12, 2020.

*Address correspondence to Samuel Barnett, M.D., Division of Sports Medicine, Department of Orthopaedic Surgery, Boston Children's Hospital, Harvard Medical School, 300 Longwood Ave., Boston, MA 02115. E-mail: Samuel.barnett@childrens.harvard.edu

(C) 2020 THE AUTHORS. Published by Elsevier Inc. on behalf of the Arthroscopy Association of North America. This is an open access article under the CC BY-NC-ND license (http://creativecommons.org/licenses/by-nc-nd/4.0/). 2666-061X/191031

https://doi.org/10.1016/j.asmr.2020.02.004 
Greater body mass index and greater preoperative pain (lower Knee Injury and Osteoarthritis Outcome Scores pain score) correlated with greater postoperative opioid use per day. There was an overprescription of opioids across all patients. Level of Evidence: Level III, case control study (therapeutic).

A nterior cruciate ligament (ACL) injuries requiring reconstruction are growing in frequency, with greater than 100,000 ACL reconstructions occurring annually in the United States. ${ }^{1}$ Health care systems, surgeons, and the wider community are more aware of the deleterious effects of prolonged and excessive opiate usage pre- and postoperatively, which has prompted a large body of research aimed at reducing the impact of this "epidemic." ${ }^{2-5}$ Opioid use has increased substantially in the past decade, due in part to emphasis on greater pain control in postsurgical patients, and prescribing practices vary widely in orthopaedic surgery, including after ACL procedures. ${ }^{6,7}$ There is a delicate balance between providing adequate analgesia and overprescribing narcotics, especially in vulnerable patient populations, such as adolescents. Illicit diversion of these medications may be a substantial contributor to significant opioid-related abuse and opioid-related mortality. ${ }^{8-10}$ A recent study found that patients younger than 25 years of age were at an increased risk of filling opioid prescriptions postoperatively. ${ }^{11}$ It is also particularly important to consider this factor in relation to outpatient/day procedures, where pain management and recovery will be largely patient-driven. In the context of these issues, however, very little literature exists on specific numbers of pills or dosages taken after common knee surgical procedures, including ACL reconstruction or ACL repair. This information is crucial to help modify prescribing patterns and developing standardized guidelines to reduce opioid-associated morbidity.

In addition, with the introduction of surgical procedures, it is crucial to understand the impact these procedures have on postoperative pain to continue safe analgesic prescribing practices. The bridge-enhanced ACL repair technique has been shown to be a safe and effective alternative to anterior cruciate ligament reconstruction (ACLR) in a small group of patients. ${ }^{12}$ The purposes of this study were to compare postoperative pain scores and opioid usage between patients undergoing a standard arthroscopic ACL reconstruction using hamstring autograft (ACLR) with those undergoing a suture repair augmented with an extracellular matrix scaffold (bridge-enhanced ACL repair) performed through an arthrotomy and to determine factors predictive of postoperative opioid use and levels of overprescription. We hypothesized that there would be no increase in the postoperative opioid requirement for the subjects undergoing bridgeenhanced ACL repair when compared with ACLR.

\section{Methods}

\section{Trial Design}

An Investigational Device Exemption (IDE; G140151) from the Food and Drug Administration and an institutional review board approval from were obtained before initiating the study. The trial was registered on ClinicalTrials.gov. All patients granted their informed consent before participating. The study was designed under the guidance of the Food and Drug Administration as an interventional, parallel assignment, nonrandomized first-in-human trial for a specific extracellular matrix scaffold designated for use in a bridge-enhanced ACL repair. For all physical examination and functional testing, the examiner was blinded to the group assignment and operative knee. All surgeries were performed at a single site by a single surgeon. Ten patients were enrolled in the interventional (bridge-enhanced ACL repair) and 10 in the control (autograft hamstring ACLR) groups. No regional anesthesia was administered. Enrollment was completed from February to October of 2015, and the patientreported outcomes and functional results have been previously reported. ${ }^{13}$

\section{Participants and Entry Criteria}

Patients aged 18 to 35 years with a complete, midsubstance ACL tear who were less than 1 month from injury and who had at least $50 \%$ of the length of the ACL attached to the tibia on their preoperative magnetic resonance imaging were eligible to enroll in the bridge-enhanced ACL repair group. As the ACL remnant is commonly removed during ACLR, and thus resorption of the torn ACL over time was not as critical, patients with a complete ACL tear who were within 3 months of injury were eligible to enroll in the ACLR group. Patients with a partial ACL tear were not eligible. Patients were excluded from either group if they had a history of previous surgery on the knee, history of previous infection in the knee, or had risk factors that might adversely affect healing. Patients were excluded if they had a displaced bucket handle tear of the medial meniscus that required repair; all other meniscal injuries were included. Patients were excluded if they had a full-thickness chondral injury, a grade III medial collateral ligament injury, a concurrent complete patellar dislocation, or an operative posterolateral corner injury. Patients in the bridgeenhanced ACL repair group also were excluded at the time of surgery if they were found to have less 
than $50 \%$ of the length of the ACL still attached to the tibial footprint.

Two hundred forty-two patients presenting with an ACL injury were screened for participation in this study. Of the 242 patients screened, 22 were enrolled in the study, of whom 2 were excluded before surgery, one due to a history of corticosteroid use not discovered in the initial enrollment meeting and the second patient elected to move to Florida for school. The primary reason for exclusion before enrollment was age (181 patients).

\section{Extracellular Matrix Scaffold}

The extracellular matrix scaffold (BEAR Scaffold) passed all biocompatibility and sterility testing. ${ }^{14-16}$ The scaffold was composed of extracellular matrix proteins, including collagen, that were obtained from bovine tissue. The scaffold measured $22 \mathrm{~mm}$ in diameter by 45 $\mathrm{mm}$ in length and was hydrophilic; able to absorb up to 5 times its weight in fluid. The scaffold was conformable to the intra-articular notch and able to fill in the irregular contours of the gap between the torn ligament ends. The safety and efficacy of the scaffold for stimulating ACL healing have been reported in preclinical studies before beginning the trial ${ }^{17-20}$ and for this firstin-human trial. ${ }^{13,21}$

\section{Surgical Techniques}

An examination under anesthesia was performed to verify that the ACL of the injured knee was deficient. A knee arthroscopy was performed and meniscal pathology addressed if necessary, and then a bridge-enhanced ACL repair was performed as previously described. ${ }^{12,13}$ To summarize in brief, $4.5-\mathrm{mm}$ tunnels were made in the femur and tibia. A 50-mm medial arthrotomy was made and a whip stitch of \#2 absorbable sutures (VICRYL; Ethicon, Somerville, NJ) was placed into the tibial stump of the torn ACL. Two \#2 non-absorbable sutures (ETHIBOND; Ethicon) were looped through the 2 center holes of a cortical button (ENDOBUTTON; Smith \& Nephew, Andover, MA) and the free ends of the suture from the tibial stump passed through the cortical button. The button carrying the nonabsorbable and absorbable sutures was passed through the femoral tunnel and engaged on the lateral femoral cortex. The \#2 nonabsorbable sutures were passed through the scaffold and then brought through the tibial tunnel. Ten cc of autologous blood was obtained from the patient via venipuncture and added to the scaffold which was then passed up along the sutures into the femoral notch. The nonabsorbable sutures were tensioned with the knee in full extension and tied over a second cortical button on the anterior tibial cortex. The absorbable sutures from the tibial stump were tied over the femoral cortical button to bring the tibial ACL stump into the scaffold and directed towards the location of the femoral insertion. The arthrotomy was closed in layers.

\section{ACL Reconstruction With ACLR}

A standard quadruple bundle hamstring autograft ACLR procedure was performed. The hamstrings were harvested through a small incision along the proximal medial tibia. A quadruple semitendinosus-gracilis graft was created and looped over a continuous-loop cortical button (ENDOBUTTON; Smith \& Nephew) for proximal fixation. A bioabsorbable interference screw (BioRCI HA; Smith \& Nephew) was used for tibial fixation through the hamstring harvest incision.

\section{Postoperative Rehabilitation: Both Groups}

For all patients, a locking hinged brace (TScope; Breg, Carlsbad, CA) was applied to limit joint range of motion between 0 to $50^{\circ}$ of knee flexion for the first 2 weeks postoperatively, from 0 to $90^{\circ}$ for the next 4 weeks unless they had a concomitant meniscal repair, in which case the brace range was restricted to 0 to $40^{\circ}$ for the first 4 weeks postoperatively before opening the brace up to 0 to $90^{\circ}$ of flexion. All patients were provided with a cold therapy unit (Iceman; DJO Global, Vista, CA) for postoperative use. Both groups followed the same standardized physical therapy protocol with range of motion restricted from 0 to $90^{\circ}$ for 6 weeks, partial weight bearing restricted for 2 weeks, then weight bearing as tolerated with crutches until 4 weeks postoperatively. Use of a functional ACL brace (CTi brace; OSSUR, Orange County, CA) was recommended from 6 to 12 weeks postoperatively.

\section{Outcome Measures}

Pain was assessed via a visual analog scale (VAS), along with simple functional outcomes including effusion and range of motion. Patient-reported outcome measures, including the International Knee Documentation Committee (IKDC) score and the Knee Injury and Osteoarthritis Outcome Scores (KOOS), were recorded at baseline and at each postoperative visit up to 2 years. The VAS is a unidimensional measure of pain intensity, consisting of a straight line $78 \mathrm{~mm}$ in length with endpoints defining opposing extremes of pain; "No pain" and "Worst pain." The subject is asked to mark his/her pain level representing the pain in their knee on that day, on the line between the endpoints. The distance between "No pain" and the subject's mark is then measured in $\mathrm{mm}$ and divided by the $78 \mathrm{~mm}$ total length to obtain the final VAS score $(0=$ no pain, $1=$ worst pain). The IKDC Subjective Score and the KOOS score consisting of 5 components, including a Pain Score, were used to assess patient-reported outcomes. $^{22-27}$ A pain medication log was provided to subjects on discharge. Subjects were asked to document the time, date, medication, and dose for each 
medication used, which was then collected and reviewed at their 2-week postoperative appointment. Additional pain medications used in the postanesthesia care unit were recorded from the medical chart. No regional anesthesia was performed. Oxycodone discharge prescriptions also were collected from the medical chart. Overprescription of opioids was calculated by subtracting the total oxycodone taken after discharge (i.e., excluding any oral opioid given as an inpatient before discharge and any other oral opioids taken by the patient that were not prescribed) from the amount prescribed to the patient. The unused rate was calculated as the amount not taken as a percentage of that which was prescribed. No refill prescriptions were filled by any patient. Morphine-equivalent dose (MED) values were calculated using the Centers for Disease Control and Prevention conversion table ${ }^{28}$ based on all opiate medication taken postoperatively.

\section{Statistical Analysis}

Subject characteristics at baseline and surgery are summarized with descriptive statistics. Differences between treatment groups were assessed using $t$ test. Mixed effects models were used to assess the association of repeated measures of outcomes with treatment (treatment $=$ bridge-enhanced ACL repair vs ACL reconstruction) over time. Models with treatment*time interaction were fitted. If the interaction term was not statistically significant, models were fitted without it. As there was no significant effect of surgical group on the postoperative opioid use, the 2 groups were pooled to determine the effect of other variables. We assessed the univariate correlation of preoperative and intraoperative variables, including age, sex, body mass index (BMI), IKDC, KOOS, tourniquet time, and meniscus repair, with postoperative opioid use, measured as the total MED and dose per day. We then included the variables that showed statistically significant correlation with postoperative opioid use in regression models, adjusted for demographics. SAS. version 9.4 (SAS Institute, Cary, NC) was used for the analysis. Statistically significant results are noted if the $P$ value is less than .05 .

\section{Results}

\section{Baseline Characteristics and Intraoperative Findings}

The baseline characteristics of both groups are shown in Table 1 and have been previously published. ${ }^{12,13}$ In summary, the 2 groups were similar in age, sex, race, and BMI. The average age of the bridge-enhanced ACL repair group was $24.1 \pm 4.9$ years and $24.6 \pm 5.5$ years in the ACLR group. The mean time from injury to surgery was significantly longer in the ACLR group (21 days vs 53 days). The numbers of patients with concomitant meniscal tears were similar between groups, as was the degree of effusion at the time of surgery.

\section{Pain Outcomes}

No patient in either group required postoperative admission, readmission, or an emergency department visit for pain issues. VAS results at 2 weeks, 6 weeks, 3 months, 6 months, 1 year, and 2 years demonstrated no difference between the 2 groups; the values in both groups decreased over time at a similar rate $(P$ value for treatment*time interaction $=.8512, P$ value for time $<.004)$, reaching a mean score at 2-years of .04 for both the bridge-enhanced ACL repair and ACLR groups. Table 2 shows pain scores from baseline to 2 years in the 2 groups.

Table 1. Baseline Characteristics

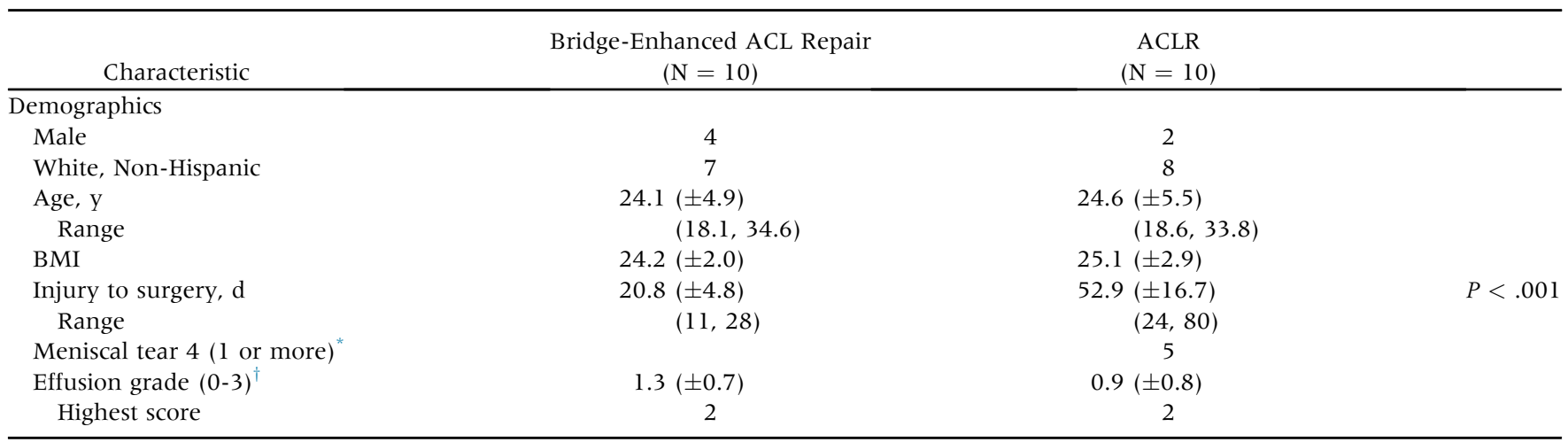

NOTE. Data presented as mean ( \pm standard deviation). Previously published with the 3-month and 2-year data for this group. ${ }^{12,1}$

ACL, anterior cruciate ligament; ACLR, anterior cruciate ligament reconstruction.

*Bridge-enhanced ACL repair: 1 lateral tear in 1 patient, 2 lateral tears in 1 patient , 1 medial tear in 2 patients. ACLR: 1 lateral tear in 3 patients,

2 lateral tears in 1 patient, 1 medial tear in 1 patient.

${ }^{\dagger} \mathrm{N}=9$ in the ACLR group. 


\section{Postoperative Opioid Consumption}

The total opiate intake measured as a MED ranged from 30 to $309 \mathrm{mg}$ (4-42 pills of oxycodone) for the ACLR group and 75 to $254 \mathrm{mg}$ (10-32 pills) for the bridge-enhanced ACL repair group in the first 2 weeks after surgery and was not significantly different between the 2 groups (Table 3, $P=.18$ ). Of this total, the amount taken as an outpatient for the ACLR group ranged from 22.5 to $277.5 \mathrm{mg}$ (337 pills of oxycodone) and 75 to $253.5 \mathrm{mg}$ (10-34 pills) MED for the BEAR group (Table $3, P=.18$ ). The average total MED consumed per day was 35.8 mg (5 pills) for the bridge-enhanced ACL repair group, and $44.2 \mathrm{mg}$ (6 pills) for patients undergoing ACLR, which was not significantly different (Table 3, $P=.29$ ).

\section{Predictors of Increased Opioid Use}

As there was no significant difference in either opioid consumption in total MED or MED per day, the 2 groups were combined in the univariate analysis for predictors. The univariate analysis revealed that greater preoperative BMI $(P=.01)$ and lower (indicating more pain) preoperative KOOS Pain Score $(P=.02)$ correlated with increased postoperative opioid use per day (Fig 1, Table 4), but no factor correlated with total MED postoperative dose. The associations with the per-day use remained statistically significant when both were included in a regression model adjusted for patient demographics. The addition of a meniscus tear requiring operative treatment at the time of ACL surgery did not impact postoperative opioid use, neither total amount or dose per day (Fig 2). The following were also not found to have an association with either measure of increased opioid consumption: age, sex, preoperative IKDC score, or total intraoperative tourniquet time.

\section{Oversupply/Overprescription}

Across both groups, the average amount prescribed was 63 pills of oxycodone ( $5 \mathrm{mg}$ each). This led to an average prescribed oversupply of oxycodone of 43 pills per patient (amount remaining after ceasing use) with a range of oversupply from 23 pills to 74 pills (Table 5).

\section{Discussion}

There was no difference in total opioid use between the 2 groups. This suggests that even though the bridgeenhanced ACL repair procedure requires an arthrotomy, this may not result in increased opioid use over the standard arthroscopic ACL reconstruction procedure. The potential postoperative pain from the arthrotomy in the bridge-enhanced ACL repair group may be mitigated by the lack of donor-site morbidity from a hamstring graft harvest site in these patients. This is important to patients and surgeons alike, particularly considering the increasing worries surrounding opioid diversion and unregulated use following surgical procedures..$^{29,30}$ Surgeons have become increasingly aware of opioid use in their patients, and the data presented here suggest no additional opioid use is needed with bridge-enhanced ACL repair (as opposed to ACL reconstruction). In addition, these data provide the first glimpse of opioid requirements for the postoperative outpatient phase of this procedure, even without the use of a nerve block, which may help contribute to standardized opioid prescribing practices for bridge-enhanced repair, as is currently being advocated for in other procedures. $^{31,32}$

There is limited evidence in the previous literature detailing opioid consumption patterns in patients undergoing an ACL reconstruction or related procedure, likely largely due to its outpatient nature and subsequent difficulty with accurate follow-up. Tepolt et al. ${ }^{33}$ were able obtain a $60 \%$ follow-up rate when using patient-reported medication logbooks and found that for those undergoing any ACL procedure, the mean number of opioid pills ( 1 pill $=5 \mathrm{mg}$ oxycodone $=7.5$ mg MED) consumed was $20.6 \pm 13.3$ (range 0-69). This equates to a $155 \pm 100 \mathrm{mg}$ MED, similar to the $187.2 \mathrm{mg}$ MED seen in the subjects treated with bridge-enhanced ACL repair and the $138.3 \mathrm{mg}$ MED seen in the subjects undergoing ACL reconstruction. Another study by Taylor et al., ${ }^{34}$ which looked specifically at opioid consumption after ACLR, found a median use of 20 pills of oxycodone, equating to $150 \mathrm{mg}$ MED. MacDonald et al. $^{35}$ compared groups undergoing single-bundle versus double-bundle ACLR and found an average oral opioid use of 39 and 49 pills in each group, respectively. However, in the study of MacDonald et al., there was no distinction made between an oral Percocet, oral Tramacet, or oral Tylenol-Codeine pill, so assuming each patient took only Tylenol-Codeine (which contains the least MED at $3 \mathrm{mg}$ MED per pill), the average use was $117.6 \mathrm{mg}$ MED for single bundle

Table 2. Pain Scores for BEAR Versus ACLR

\begin{tabular}{|c|c|c|c|c|c|}
\hline \multirow[b]{2}{*}{ Outcome } & \multicolumn{2}{|c|}{$\operatorname{BEAR}(\mathrm{n}=10)$} & \multicolumn{2}{|c|}{$\operatorname{ACLR}(\mathrm{n}=10)$} & \multirow[b]{2}{*}{$P$ Value } \\
\hline & $\mathrm{N}$ & Mean (SD) & $\mathrm{N}$ & Mean (SD) & \\
\hline \multicolumn{6}{|c|}{ Visual analog scale } \\
\hline $2 \mathrm{wk}$ & 10 & $0.59(0.28)$ & 10 & $0.72(0.23)$ & .2835 \\
\hline $6 \mathrm{wk}$ & 10 & $0.27(0.24)$ & 10 & $0.17(0.16)$ & .2841 \\
\hline $3 \mathrm{mo}(12 \mathrm{wk})$ & 10 & $0.19(0.15)$ & 10 & $0.14(0.23)$ & .5472 \\
\hline $6 \mathrm{mo}(26 \mathrm{wk})$ & 10 & $0.20(0.24)$ & 9 & $0.15(0.16)$ & .5876 \\
\hline 1 y (52 wk) & 10 & $0.11(0.14)$ & 8 & $0.18(0.27)$ & .4631 \\
\hline 2 y (104 wk) & 8 & $0.04(0.11)$ & 7 & $0.04(0.08)$ & .9586 \\
\hline
\end{tabular}

$\mathrm{ACL}$, anterior cruciate ligament; ACLR, anterior cruciate ligament reconstruction; BEAR, bridge-enhanced ACL repair; SD, standard deviation.

${ }^{*} P$ value for $t$ test, Satterthwaite $P$ if unequal variance. 
Table 3. Opioid Use for Bridge-Enhanced ACL Repair Versus ACLR

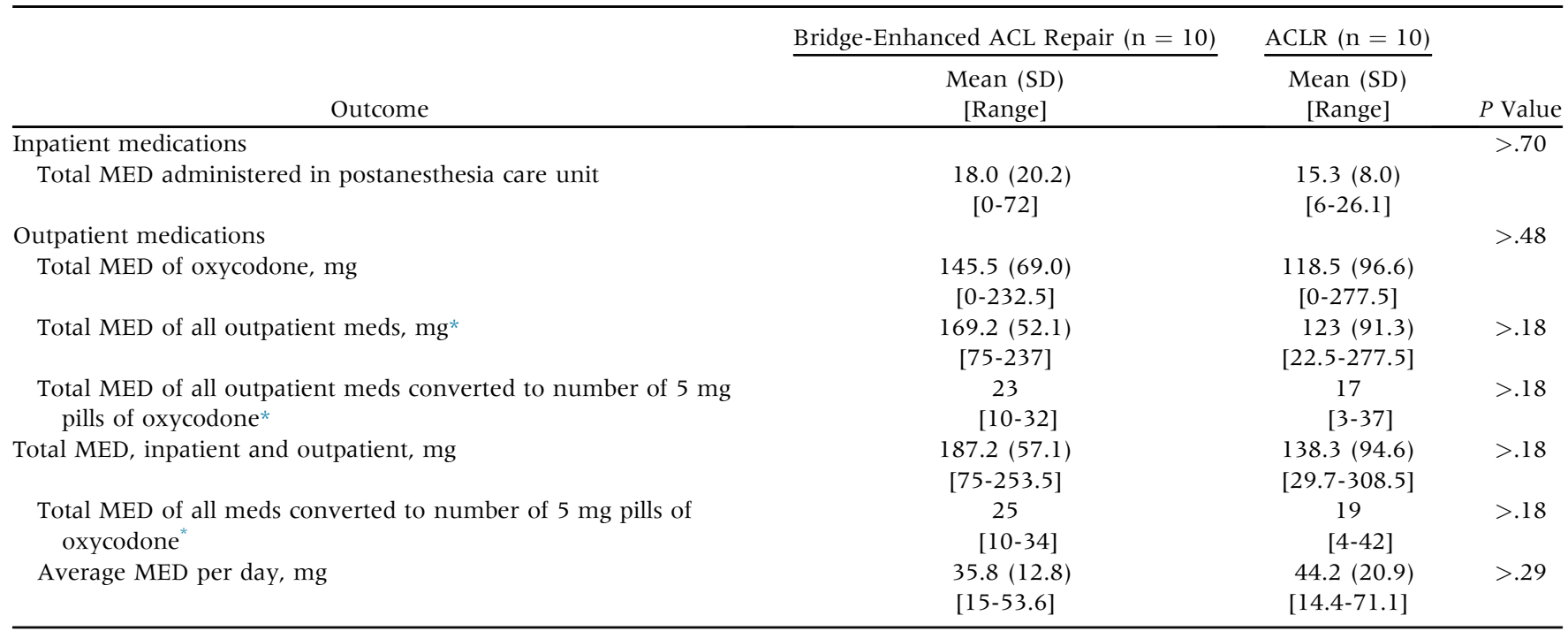

$\mathrm{CL}$, anterior cruciate ligament; ACLR, anterior cruciate ligament reconstruction; BEAR, bridge-enhanced ACL repair; MED, morphineequivalent dose; SD, standard deviation.

*Includes all outpatient opioids consumed (as documented on patient pain medication log), including those not prescribed directly by our institution.

and $147 \mathrm{mg}$ MED for double bundle ACLR. Although limited, this evidence is consistent with our small study. Of note, patients in our study were not given pre- or postoperative nerve blocks, which have been shown to reduce postoperative opioid use. ${ }^{36,37}$

Given the similarity between the 2 groups in terms of postoperative opioid use, we combined the 2 groups and then further analyzed both preoperative and intraoperative markers to predict postoperative opioid use in all patients. We demonstrated that patients with a greater BMI and those that had more pain in their injured knee preoperatively (indicated by a lower preoperative KOOS pain score) took more opioid medication postoperatively. This is consistent with Tepolt et al., ${ }^{33}$ who showed via multivariate analysis that a patient's weight was predictive of postoperative opioid consumption. It has also been shown previously that filling opioid prescriptions preoperatively increased demand for postoperative opioid use after ACL reconstruction. ${ }^{1,38}$ A related factor to this is preoperative pain and pain tolerance, in which we have demonstrated that those with a greater baseline preoperative pain took more opioids postoperatively. This may indicate the utility in obtaining preoperative pain scales in patients undergoing ACLR or a similar procedure to help adapt postoperative clinical care. This is an important clinical step in tailoring opioid prescription for patients known to have greater tendency to either
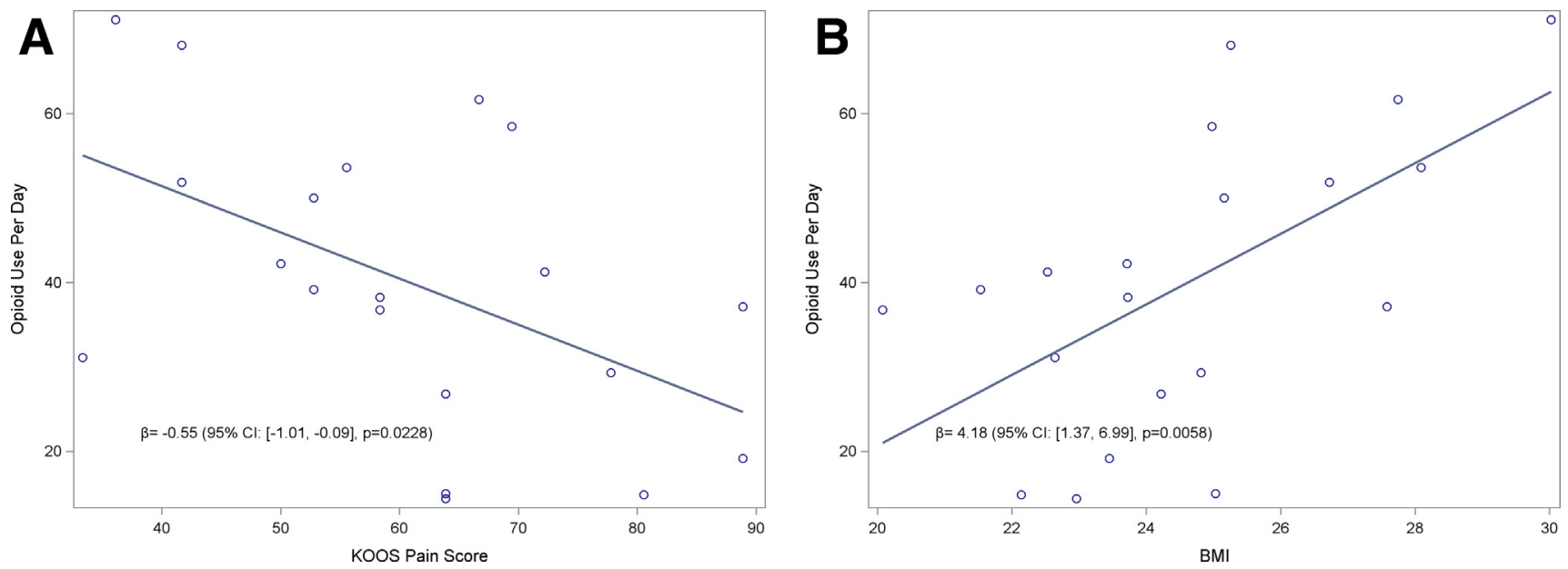

Fig 1. Correlation of preoperative KOOS Pain Score (A) and BMI (B) with postoperative opioid use. Note that a greater KOOS Pain Score is indicative of less pain reported. KOOS, Knee Injury and Osteoarthritis Outcome Scores; BMI, body mass index. 
Table 4. Correlation of Preoperative and Intraoperative Variables with Postoperative Opioid Use

\begin{tabular}{lccr}
\hline & \multicolumn{2}{c}{ MED per day } & \multicolumn{2}{c}{ Total MED } \\
\cline { 2 - 4 } \multicolumn{1}{c}{ Variable } & Correlation Coefficient & $P$ Value & Correlation Coefficient \\
\hline Age & -0.3613 & .1176 & -0.1883 \\
BMI & $\mathbf{0 . 5 9 3 4}$ & $\mathbf{. 0 0 5 8}$ & 0.2386 \\
Baseline IKDC & -0.2037 & .3890 & -0.2259 \\
Baseline KOOS Symptoms & -0.0344 & .8855 & .3109 \\
Baseline KOOS Pain & $-\mathbf{0 . 5 0 6 2}$ & $\mathbf{. 0 2 2 8}$ & .3382 \\
Baseline KOOS ADLs & -0.3542 & .1255 & .0 .2236 \\
Baseline KOOS Sports & -0.2021 & .3930 & -0.2379 \\
Baseline KOOS QOL & -0.3305 & .1547 & -0.2872 \\
Tourniquet Time, min & -0.4184 & .0664 & -0.3829 \\
\hline
\end{tabular}

NOTE. Boldface indicates statistical significance.

ADL, activity of daily living; BMI, body mass index; IKDC, International Knee Documentation Committee; KOOS, Knee Injury and Osteoarthritis Outcome Scores; MED, morphine-equivalent dose; QOL, quality of life.

require more opioid for better pain control, or to warrant more due to biological make-up. The ultimate aim is to reduce longer-term morbidity associated with excess use, and contribute to applicable opioid prescribing guidelines. In addition, we demonstrated that when meniscus repair is performed concurrently with an ACL reconstruction or bridge-enhanced ACL repair, there was no impact on postoperative opioid use. Beck et al. ${ }^{39}$ showed a similar finding in which the addition of meniscus repair or partial meniscectomy did not affect postoperative opioid use. Patients were excluded from our study if they had a displaced bucket-handle tear of the medial meniscus, so this particular type of meniscus injury cannot be commented on. With consideration of this limitation, it would seem that the ACL reconstruction/repair may be the primary factor dictating the pain response and subsequent opioid use in these patients.

Our study adds to the previous literature in regards to the overprescription of opioid medication in postsurgical orthopaedic patients, even in the setting of no pre- or postoperative nerve block. We demonstrated a greater than $70 \%$ unused opiate rate across the 20 patients enrolled into this clinical trial, equating to an average oversupply of 46 pills of oxycodone. This was consistent with a recently published study that reported a $68 \%$ unused opioid rate in adolescents and young adults undergoing knee arthroscopy and related procedures. ${ }^{33}$ Kim et al. ${ }^{40}$ evaluated opioid use after upperextremity surgical procedures and found a $66 \%$ unused rate. Sabatino et al. ${ }^{6}$ looked at adult patients undergoing 5 common elective orthopedic procedures (total hip arthroplasty, total knee arthroplasty, endoscopic carpal tunnel release, arthroscopic rotator cuff repair and lumbar decompression) and demonstrated that $61 \%$ of patients had excess opioid medication remaining, accounting to 43,216 unused pills. These significant incongruence rates between opioids prescribed and opioids consumed for common orthopaedic procedures opens an opportunity for clinicians to begin to contribute to the reduction of opioids in the community. This would be a positive step in restricting
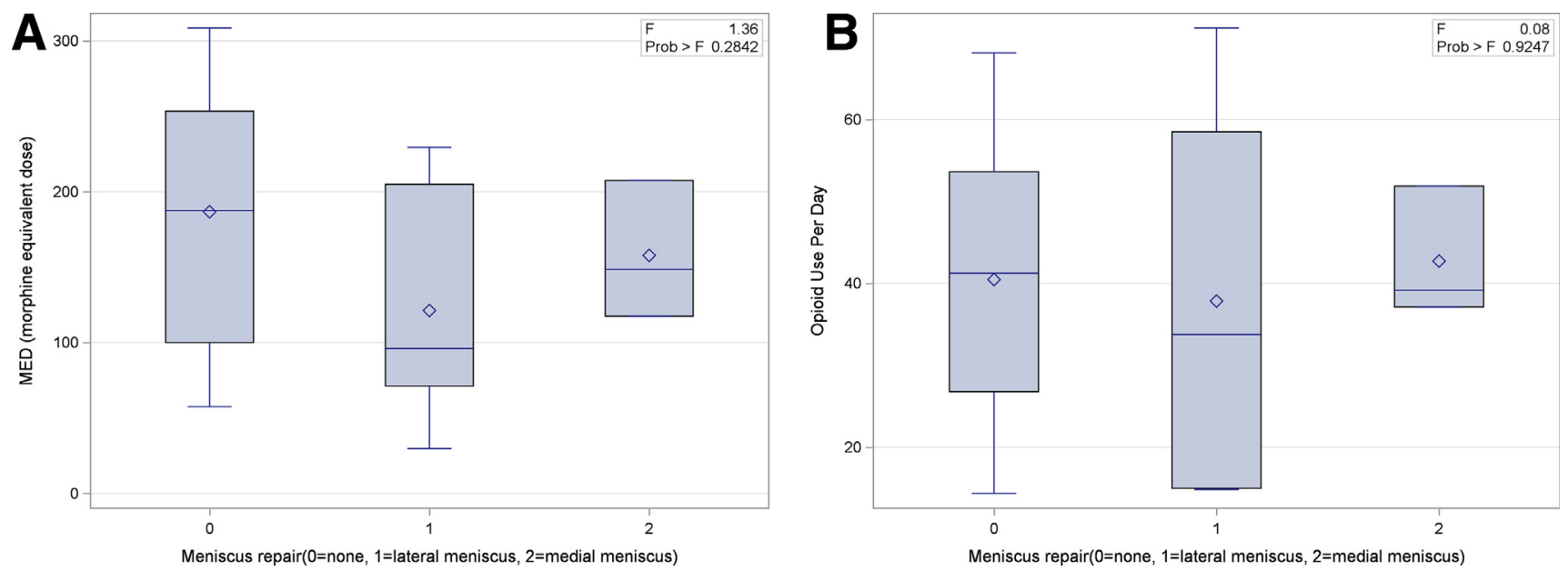

Fig 2. Comparison of total MED (A) and opioid use per day (B) in patients with and without a meniscal repair. MED, morphineequivalent dose. 
Table 5. Oxycodone Prescription Versus Consumption

\begin{tabular}{|c|c|c|c|}
\hline & $\underline{\text { Bridge-Enhanced ACL Repair }(\mathrm{n}=10)}$ & ACL Reconstruction $(\mathrm{n}=9)$ & \\
\hline Outcome & Mean (SD) & Mean (SD) & $P$ Value \\
\hline Total no. oxycodone pills prescribed & $\begin{array}{c}64.8(10.1) \\
{[60-84]}\end{array}$ & $\begin{array}{l}60(0) \\
{[60-60]}\end{array}$ & $>.17$ \\
\hline Total no. oxycodone pills taken & $\begin{array}{c}19.4(9.2) \\
{[0-31]}\end{array}$ & $\begin{array}{c}13.8(11.9) \\
{[0-37]}\end{array}$ & $>.27$ \\
\hline Total no. oxycodone pills left over & $\begin{array}{c}45.4(16.5) \\
{[29-74]}\end{array}$ & $\begin{array}{c}46.2(11.9) \\
{[23-60]}\end{array}$ & $>.90$ \\
\hline
\end{tabular}

ACL, anterior cruciate ligament; SD, standard deviation.

potential for illicit diversion of these medications, which is aggravated by the lack of knowledge about safe disposal practice, which has previously been reported. $6,33,39,41$

The current analysis directs the need for further research on pain outcomes and medication use following the bridge-enhanced ACL repair technique. It would further be of use to look at opioid use in conjunction with the commonly used femoral nerve or adductor canal block in patients undergoing the bridgeenhanced ACL repair procedure, to see if corresponding reductions in opioid use are seen. Our study continues to add evidence for the substantial over prescription of opioids to both adolescents and adults for postoperative pain after orthopaedic procedures. We have subsequently made systematic changes to our prescribing practices at our institution and encourage others to follow.

\section{Limitations}

There are several limitations of our current study. It was constructed primarily for the analysis of safety outcomes, and only enrolled small numbers. Thus, we acknowledge the possibility that this study is underpowered, with only 10 subjects in each treatment group. A priori power calculation was not performed based on opioid consumption because it is not the primary outcome as planned. The trial and sample size calculation were designed to report adverse events and ensure safety. However, ad hoc power analysis applied to studies with negative findings is unreliable because the calculation of power based on observed negative results will always lead to low power. ${ }^{42,43}$ Further, attributing the negative results to low power is problematic. Instead, as recommended by current literature, $^{42,43}$ we examined the confidence intervals $(95 \%$ $\mathrm{CI}$ ) of our estimates. For average opioid use per day, the mean difference between groups (ACLR - BEAR) is 8.3 mg ( 1.1 pills) $(95 \% \mathrm{CI}-7.9$ to $24.6, P=.30)$. First, this means we should not rule out the possibility that there is no difference between groups because zero in contained in the $95 \%$ CI. Second, it is possible that the daily opioid use in the ACLR group is $7.9 \mathrm{mg}$ ( 1.1 pills) lower than the BEAR group. Conversely, the data are also consistent with the possibility that daily use is $\mathbf{2 4 . 6}$ mg greater in ACLR than BEAR. This translates into patients undergoing ACLR taking 3.3 more pills per day than patients undergoing BEAR. A similar argument can be made for total MED, for which the mean difference is $-48.8 \mathrm{mg}$ ( 6.5 pills) ( $95 \% \mathrm{CI}-122.2$ to 24.6 , $P=.18)$. Therefore, we concluded that opiate intake is likely not different between groups. Detailed postoperative opioid consumption in terms of actual pills consumed following outpatient orthopaedic procedures is also scarce in the literature and so even small numbers adds weight to current knowledge, especially in a younger population. Quantities of opioid consumption were also patient reported and therefore it is important to acknowledge that this could lead to an underestimate of intake in patients who may not have accurately completed their medication logs. However, all patients completed their logs and provided evidence of this, removing the issue of selection bias. Finally, all patients were operated on by a single surgeon at a single institution, which may reduce variability, but may also make our conclusions less generalizable.

\section{Conclusions}

Total overall opiate intake was not different between the patients undergoing BEAR through an arthrotomy and those undergoing arthroscopic ACLR. Both groups had similar pain scores from 2 weeks to 2 years postoperatively. Greater BMI and greater preoperative pain (lower KOOS pain score) correlated with greater postoperative opioid use per day. There was an overprescription of opioids across all patients.

\section{Acknowledgments}

We acknowledge the significant contributions of the clinical trial team, including Bethany Trainor, and Shanika Coney. We also acknowledge the contributions of our medical safety monitoring team of Joseph DeAngelis, Peter Nigrovic, and Carolyn Hettrich; our data monitors Maggie Malsch, Meghan Fitzgerald, and Erica Denhoff; as well as the clinical care team for the trial patients, including Kathryn Ackerman, Alyssa Aguiar, Judd Allen, Michael Beasley, Jennifer Beck, Dennis Borg, Jeff Brodeur, Stephanie Burgess, Melissa Christino, Sarah Collins, Gianmichel Corrado, Sara 
Carpenito, Corey Dawkins, Pierre D'Hemecourt, Jon Ferguson, Michele Flannery, Casey Gavin, Ellen Geminiani, Stacey Gigante, Annie Griffin, Emily Hanson, Elspeth Hart, Jackie Hastings, Pamela Horne-Goffigan, Christine Gonzalez, Meghan Keating, Ata Kiapour, Elizabeth KillKelly, Elizabeth Kramer, Pamela Lang, Hayley Lough, Chaimae Martin, Michael McClincy, William Meehan, Ariana Moccia, Jen Morse, Mariah Mullen, Stacey Murphy, Emily Niu, Michael O'Brien, Nikolas Paschos, Katrina Plavetsky, Bridget Quinn, Shannon Savage, Edward Schleyer, Benjamin Shore, Cynthia Stein, Andrea Stracciolini, Dai Sugimoto, Dylan Taylor, Ashleigh Thorogood, Kevin Wenner, Brianna Quintiliani, and Natasha Trentacosta. We also thank the perioperative and operating room staff and the members of the Department of Anesthesia, who were extremely helpful in developing the perioperative and intraoperative protocols. We also acknowledge the efforts of other scaffold manufacturing team members, including Gabe Perrone, Gordon Roberts, Doris Peterkin, and Jakob Sieker. We are also grateful for the study design guidance provided by the Division of Orthopaedic Devices at the Center for Devices and Radiological Health at the U.S. Food and Drug Administration under the guidance of Laurence Coyne and Mark Melkerson, particularly the efforts of Casey Hanley, Peter Hudson, Jemin Dedania, Pooja Panigrahi, and Neil Barkin. We are also especially grateful to the patients and their families who participated in this study; their willingness to participate in research that may help others in the future inspires all of us.

\section{References}

1. Buller LT, Best MJ, Baraga MG, Kaplan LD. Trends in anterior cruciate ligament reconstruction in the United States. Orthop J Sports Med 2015;3:2325967114563664.

2. Frieden TR, Houry D. Reducing the risks of relief-The CDC Opioid-Prescribing Guideline. $N$ Engl J Med 2016;374:1501-1504.

3. Seth P, Scholl L, Rudd RA, Bacon S. Overdose deaths involving opioids, cocaine, and psychostimulants-United States, 2015-2016. MMWR Morb Mortal Wkly Rep 2018;67: 349-358.

4. American Academy of Orthopaedic Surgeons. Opioid use, misuse, and abuse in orthopaedic practice, https://Www. aaos.org/uploadedFiles/PreProduction/About/Opinion_ Statements/advistmt/1045 Opioid Use, Misuse, and Abuse in Practice.pdf. Accessed September 2, 2019.

5. Reid DBC, Shah KN, Shapiro BH, Ruddell JH, Akelman E, Daniels AH. Mandatory prescription limits and opioid utilization following orthopaedic surgery. J Bone Joint Surg Am 2019;101:e43.

6. Sabatino MJ, Kunkel ST, Ramkumar DB, Keeney BJ, Jevsevar DS. Excess opioid medication and variation in prescribing patterns following common orthopaedic procedures. J Bone Joint Surg Am 2018;100:180-188.
7. Secrist ES, Freedman KB, Ciccotti MG, Mazur DW, Hammoud S. Pain management after outpatient anterior cruciate ligament reconstruction: A systematic review of randomized controlled trials. Am J Sports Med 2016;44: 2435-2447.

8. Gaither JR, Leventhal JM, Ryan SA, Camenga DR. National trends in hospitalizations for opioid poisonings among children and adolescents, 1997 to 2012. JAMA Pediatr 2016;170:1195-1201.

9. Allen JD, Casavant MJ, Spiller HA, Chounthirath T, Hodges NL, Smith GA. Prescription opioid exposures among children and adolescents in the United States: 2000-2015. Pediatrics 2017;139(4).

10. Steiner SRH, Cancienne JM, Werner BC. Narcotics and knee arthroscopy: Trends in use and factors associated with prolonged use and postoperative complications. Arthroscopy 2018;34:1931-1939.

11. Anthony CA, Westermann RW, Bedard N, et al. Opioid demand before and after anterior cruciate ligament reconstruction. Am J Sports Med 2017;45:3098-3103.

12. Murray MM, Flutie BM, Kalish LA, et al. The bridgeenhanced anterior cruciate ligament repair (BEAR) procedure: An early feasibility cohort study. Orthop J Sports Med 2016;4:2325967116672176.

13. Murray MM, Kalish LA, Fleming BC, et al. Bridgeenhanced anterior cruciate ligament repair: Two-year results of a first-in-human study. Orthop J Sports Med 2019:7:232596711882435.

14. Proffen BL, Perrone GS, Fleming BC, et al. Effect of lowtemperature ethylene oxide and electron beam sterilization on the in vitro and in vivo function of reconstituted extracellular matrix-derived scaffolds. J Biomater Appl 2015;30:435-449.

15. Proffen BL, Perrone GS, Fleming BC, et al. Electron beam sterilization does not have a detrimental effect on the ability of extracellular matrix scaffolds to support in vivo ligament healing. J Orthop Res 2015;33:1015-1023.

16. Proffen B, Perrone G, Roberts G, Murray M. Bridgeenhanced ACL repair: A review of the science and the pathway through FDA investigational device approval. Ann Biomed Eng 2015;43:805-818.

17. Yoshida R, Vavken P, Murray MM. Decellularization of bovine anterior cruciate ligament tissues minimizes immunogenic reactions to alpha-gal epitopes by human peripheral blood mononuclear cells. Knee 2012;19: 672-675.

18. Murray MM, Magarian E, Zurakowski D, Fleming BC. Bone-to-bone fixation enhances functional healing of the porcine anterior cruciate ligament. Arthroscopy 2010;26: S49-S57 (suppl).

19. Joshi SM, Mastrangelo AN, Magarian EM, Fleming BC, Murray MM. Collagen-platelet composite enhances biomechanical and histologic healing of the porcine anterior cruciate ligament. Am J Sports Med 2009;37: 2401-2410.

20. Magarian EM, Vavken P, Connolly SA, Mastrangelo AN, Murray MM. Safety of intra-articular use of atelocollagen for enhanced tissue repair. Open Orthop J 2012;6:231-238.

21. Murray MM, Kiapour AM, Kalish LA, et al. Predictors of healing ligament size and magnetic resonance signal 
intensity at 6 months after bridge-enhanced anterior cruciate ligament repair. Am J Sports Med 2019: 363546519836087.

22. Irrgang JJ, Anderson AF, Boland AL, et al. Development and validation of the international knee documentation committee subjective knee form. Am J Sports Med 200 1;29: 600-613.

23. Irrgang JJ, Anderson AF, Boland AL, et al. Responsiveness of the International Knee Documentation Committee Subjective Knee Form. Am J Sports Med 2006;34: 1567-1573.

24. Paradowski PT, Bergman S, Sunden-Lundius A, Lohmander LS, Roos EM. Knee complaints vary with age and gender in the adult population. Population-based reference data for the Knee injury and Osteoarthritis Outcome Score (KOOS). BMC Musculoskelet Disord 2006;7:38.

25. Rossi MJ, Lubowitz JH, Guttmann D. Development and validation of the International Knee Documentation Committee Subjective Knee Form. Am J Sports Med 2002;30:152.

26. Lingard EA, Katz JN, Wright RJ, Wright EA, Sledge CB, Kinemax Outcomes Group. Validity and responsiveness of the Knee Society Clinical Rating System in comparison with the SF-36 and WOMAC. J Bone Joint Surg Am 2001;83-A:1856-1864.

27. Roos EM, Lohmander LS. The Knee injury and Osteoarthritis Outcome Score (KOOS): from joint injury to osteoarthritis. Health Qual Life Outcomes 2003;1:64.

28. Centers for Disease Control and Prevention. Calculating total daily dose of opioids for safer dosage [updated November 10th, 2018], https://www.cdc.gov/drugoverdose/pdf/ calculating_total_daily_dose-a.pdf. Accessed February 1, 2019.

29. Manchikanti L, Helm S II, Fellows B, et al. Opioid epidemic in the United States. Pain Physician 2012;15: ES9-ES38 (suppl 3).

30. Trasolini NA, McKnight BM, Dorr LD. The opioid crisis and the orthopedic surgeon. J Arthroplasty 2018;33: 3379-3382.el.

31. Bedard NA, Sierra RJ, Mabry T. Opioids after orthopaedic surgery: There is a need for universal prescribing recommendations: Commentary on an article by Matthew J. Sabatino, MD, MS, et al.: "Excess Opioid Medication and Variation in Prescribing Patterns Following Common Orthopaedic Procedures. ". J Bone Joint Surg Am 2018;100:e17.

32. Scully RE, Schoenfeld AJ, Jiang W, et al. Defining optimal length of opioid pain medication prescription after common surgical procedures. JAMA Surg 2018;153:37-43.

33. Tepolt FA, Bido J, Burgess S, Micheli LJ, Kocher MS. Opioid overprescription after knee arthroscopy and related surgery in adolescents and young adults. Arthroscopy 2018;34:3236-3243.

34. Taylor N, Frick S, Killilea S, Dugan-Frost T, Solodiuk J. Opioid use in children and adolescents after anterior cruciate ligament repair. J Healthc Qual 2018;40:97-102.

35. Macdonald SA, Heard SM, Hiemstra LA, Buchko GM, Kerslake S, Sasyniuk TM. A comparison of pain scores and medication use in patients undergoing single-bundle or double-bundle anterior cruciate ligament reconstruction. Can J Surg 2014;57:E98-E104.

36. Abdallah FW, Whelan DB, Chan VW, et al. Adductor canal block provides noninferior analgesia and superior quadriceps strength compared with femoral nerve block in anterior cruciate ligament reconstruction. Anesthesiology 2016;124:1053-1064.

37. Vorobeichik L, Brull R, Joshi GP, Abdallah FW. Evidence basis for regional anesthesia in ambulatory anterior cruciate ligament reconstruction: Part I-femoral nerve block, Anesth Analg 2019;128:58-65.

38. Rao AG, Chan PH, Prentice HA, Paxton EW, Funahashi TT, Maletis GB. Risk factors for opioid use after anterior cruciate ligament reconstruction. Am J Sports Med 2019:2130-2137.

39. Beck PR, Nho SJ, Balin J, et al. Postoperative pain management after anterior cruciate ligament reconstruction. J Knee Surg 2004;17:18-23.

40. Kim N, Matzon JL, Abboudi J, et al. A prospective evaluation of opioid utilization after upper-extremity surgical procedures: Identifying consumption patterns and determining prescribing guidelines. J Bone Joint Surg Am 2016;98:e89.

41. McCauley JL, Back SE, Brady KT. Pilot of a brief, webbased educational intervention targeting safe storage and disposal of prescription opioids. Addict Behav 2013;38: 2230-2235.

42. Goodman SN, Berlin JA. The use of predicted confidence intervals when planning experiments and the misuse of power when interpreting results. Ann Intern Med 1994;121:200-206.

43. Levine M, Ensom MH. Post hoc power analysis: An idea whose time has passed? Pharmacotherapy 2001;21: 405-409. 